\title{
A host mobility support with adaptive network selection method in hybrid wireless environment
}

\begin{abstract}
The B3G or 4G of cellular/wireless communications network is expected to be purely IPbased and consist of heterogeneous access networks from $3 \mathrm{G}$ cellular, WiFi, WiMAX and a converged core network. To sup-port the mobile host in the hybrid overlay wireless, a crosslayer vertical handoff control method has been proposed. Implemented architecture is composed of four part; Connection Profile Manager (CPM), Net-work Access Assistance (NAA), Neuro-Fuzzy Decision Engine (NFDE), and Peer-bind Connection Manager (PCM). The most effective connection is selected based on the attributes from the multi-layer platform that provided handoff decision component which is respon-sible for handoff decision. The proposed network selection algorithm is based on hybrid neuro-fuzzy concept with low packet loss and latency. We have analyzed our implemented model on different scena-rios. Results of the experiment indicate the advantages of the proposed scheme.
\end{abstract}

Keyword: Handoff; mobility management; neuro-fuzzy; wireless network 